\title{
CONTRAST-ENHANCED NANOFOCUS COMPUTED TOMOGRAPHY IMAGES THE CARTILAGE SUBTISSUE ARCHITECTURE IN THREE DIMENSIONS
}

\author{
Greet Kerckhofs ${ }^{1,2,3, *}$, Julie Sainz ${ }^{4}$, Martine Wevers ${ }^{3}$, Tom Van de Putte ${ }^{4}$ and Jan Schrooten ${ }^{2,3}$ \\ ${ }^{1}$ Biomechanics Research Unit, Université de Liège, Liège, Belgium \\ ${ }^{2}$ Prometheus, Division of Skeletal Tissue Engineering Leuven, KU Leuven, Leuven, Belgium \\ ${ }^{3}$ Department of Metallurgy and Materials Engineering, KU Leuven, Heverlee, Belgium \\ ${ }^{4}$ TiGenix NV, Leuven, Belgium
}

\begin{abstract}
We describe a non-destructive imaging method, named contrast-enhanced nanofocus X-ray computed tomography (CE-nanoCT), that permits simultaneously imaging and quantifying in $3 \mathrm{D}$ the (sub)tissue architecture and (biochemical) composition of cartilage and bone in small animal models at a novel contrast and spatial resolution. To demonstrate the potential of this novel methodology, a newborn mouse was scanned using CE-nanoCT. This allowed simultaneously visualising the bone and cartilage structure much like the traditional alcian blue-alizarin red skeletal stain. Additionally, it enabled a 3D visualisation at such a high spatial image resolution that internal, microscale structures could be digitally dissected and evaluated for size, structure and composition. Ex vivo treatment with papain, that is known to specifically remove the noncalcified cartilage layer but keep the calcified cartilage intact, proved CE-nanoCT to be applicable to visualise the subdivisions within the hyaline cartilage of the articular joint of mice. The quantitative power of CE-nanoCT in vivo was evaluated using a mouse model for osteoarthritis (OA), where OA-like cartilage lesions are induced by meniscus destabilisation surgery. The thickness of both the non-calcified and calcified cartilage layer in the knee joint of such mice was visualised and quantified in $3 \mathrm{D}$ and compared to unaffected mice. Finally, to show that different forms of cartilage and tissue combinations can be distinguished using CE-nanoCT, different cartilaginous body parts of the mouse were imaged. In conclusion, $\mathrm{CE}-$ nanoCT can provide novel insights in preclinical research by quantifying in a non-destructive $3 \mathrm{D}$ manner pathological differences, in particular in developing mice, newborns or adults.
\end{abstract}

Keywords: Contrast-enhanced nanofocus computed tomography (CE-nanoCT); 3D cartilage imaging; cartilage sub-architecture; small animal 3D imaging.

*Address for correspondence:

Dr. Greet Kerckhofs

Prometheus, Division for Skeletal Tissue Engineering

KU Leuven Onderwijs en Navorsing $1(+8)$

Herestraat 49 - PB813, B-3000 Leuven, Belgium

Telephone Number: (+32) 16346189

Fax Number: (+32) 16321990

E-mail: greet.kerckhofs@mtm.kuleuven.be

\section{Introduction}

Small animal model quantification is key to understand the functionality (Botter et al., 2008) and pathophysiology of degenerative joint diseases such as osteoarthritis (OA) (Lyons, 2005), as well as other diseases where the tight interplay between the cartilaginous and bony counterparts has been distorted (e.g. craniosynostosis, achondroplasia). Currently, preclinical efficacy of novel therapies for bone and cartilage repair in small animal models or the skeletal changes during normal or aberrant foetal and postnatal development is commonly assessed by immunohistochemistry and whole mount skeletal staining (Nagy et al., 2003). They have a high discriminative power but are destructive, labour intensive, and, in standard settings, only provide 2D data. Fluorescence-based imaging techniques, such as confocal microscopy (Maye et al., 2011), optical projection tomography (OPT) (Sharpe et al., 2002) or selective plane illumination microscopy (SPIM) (Huisken et al., 2004) are alternatives which are commonly used to image gene and protein expression during developmental processes. However, for high contrast and high resolution imaging these techniques require transparent or semi-transparent samples and/or are limited to small specimens $(\sim \mathrm{mm})$.

For 3D imaging, EPIC-CT, i.e. equilibrium partitioning of an ionic contrast agent via microfocus X-ray computed tomography (microCT), has been reported to be highly applicable for simultaneous imaging and quantification of bone and cartilage structure and composition in preclinical research on small animal models (Kotwal et al., 2012; Liang et al., 2011; Palmer et al., 2006; Piscaer et al., 2008; Xie et al., 2010; Xie et al., 2009). But the limited spatial image and/or contrast resolution of EPICCT limits its use in small model organisms such as the mouse, where e.g. the thickness of the hyaline cartilage can be down to $30 \mu \mathrm{m}$ (Kotwal et al., 2012), which is nearly 10 -fold thinner than in the rat and approximately 50-fold thinner than in man. This inability of EPIC-CT to distinguish the architecture and composition of calcified versus non-calcified cartilage hampers its use to study joint diseases such as OA in small animal models, where a loss of sGAGs, fibrillations or finally full-thickness loss of non-calcified cartilage and damage of the calcified cartilage are all important features of the disease that need to be documented.

In this study, we introduce an improved methodology compared to EPIC-CT, named contrast-enhanced nanofocus X-ray computed tomography (CE-nanoCT). We 
have shown that this technique permits for the first time imaging the subtissue architecture of cartilage and bone in different body parts of mouse models in one single scan, and this at a high contrast and spatial image resolution and without destructive, labour-intensive sample preparation. High contrast differentiation between bone and cartilage, as well as other soft tissues, is achieved by the use of an anionic contrast agent (i.e. Hexabrix ${ }^{\circledR}$ ). When combined with the high resolution of nanoCT, this provides the necessary spatial image resolution to assess in 3D structure and quality of calcified and non-calcified cartilage and bone in the course of one single scan. This was proven on a mouse newborn, knee joints of mice induced with OA, and different mouse body parts.

\section{Materials and Methods}

\section{Animal models}

Animal experiments were performed in accordance with the Belgian legislation under the national authorisation number LA1210530 and were approved by the Ethical Committee of the Faculty of Biomedical Sciences of the University of Leuven.

Mouse newborn

A CD1 newborn pup (E18.5) was sacrificed by suffocation with $\mathrm{CO}_{2}$.

Mouse tissue samples

The trachea, patella, paw and several mouse knee joints were dissected from adult (Rj:NMRI-Foxn $1^{\text {nu }} /$ Foxn $^{\text {nu }}{ }^{\text {}}$ ) mice. The knee joint capsules were dislocated to expose the cartilage layers of the tibial plateau and the femoral condyles. Knee joints were left untreated or were treated ex vivo with papain from papaya latex (Sigma Aldrich BVBA, Diegem, Belgium) to enzymatically digest the noncalcified cartilage layer (treatment: papain $1 \%$ - cysteine $\mathrm{HCl}$ for $3 \mathrm{~d}$ at $60{ }^{\circ} \mathrm{C}$ ) according to the protocol described by Hughes et al. (2005).

Other knee joints were harvested from mice that were sham operated (Sham) or underwent a medial meniscus destabilisation procedure (DMM), and were sacrificed 8 weeks later (Glasson et al., 2007). Again, the knee joint capsule was dislocated to expose the cartilage layers of the tibial plateau and the femoral condyles. We have worked with the nude mouse model to assess the effect of DMM on cartilage structure and composition, because it allows testing of a cellular therapy in the mouse using human cells (on-going work).

The tissues and knee joints were processed for nanoCT imaging after which they were paraffin embedded for histological analyses.

\section{Contrast-enhanced nanofocus computed tomography (CE-nanoCT)}

NanoCT imaging uses a nanofocal spot X-ray tube, combined with high geometric magnification, and hence differs from conventional medical CT scanning and microCT in its ability to resolve details as small as a few hundreds of $\mathrm{nm}$ in size (Sasov, 2004). The CT system applied in this study was a Phoenix NanoTom $\mathrm{S}(G E$ Sensing \& Inspection Technologies GmbH, Wunstorf, Germany) with a $180 \mathrm{kV} / 15 \mathrm{~W}$ high-performance nanofocus X-ray tube. It was equipped with a tungsten target and was operated, for all scans, at a voltage of $60 \mathrm{kV}$ and a current of $140 \mu \mathrm{A}$. A $1 \mathrm{~mm}$ filter of aluminium was used. The exposure time was $500 \mathrm{~ms}$ and a frame averaging of 1 and image skip of 0 was applied, resulting in a scanning time of $20 \mathrm{~min}$. Because of the height of the mouse newborn being 4-5 times larger than its width and since the highest possible spatial image resolution was desired, 5 scans were made to capture the full newborn, resulting in a scanning time of $100 \mathrm{~min}$. The reconstructed images of the mouse newborn had an isotropic voxel size of $4.5 \mu \mathrm{m}$. The mouse paw and trachea were imaged at an isotropic voxel size of $3 \mu \mathrm{m}$. For the patella and all the knee joints, the isotropic voxel size was $2 \mu \mathrm{m}$.

The contrast agent used in this study was an injectable solution of ioxaglate meglumine $39.3 \%$ and ioxaglate sodium $19.6 \%$ (Hexabrix ${ }^{\circledR}$ 320, Guerbet Nederland BV, Gorinchem, The Netherlands). This is a radio-opaque dye, which contains ioxaglate, a radio-opaque negatively charged ionic iodinated dimer. The negatively charged ioxaglate will be locally repulsed by the negative fixed charge density of the cartilage, which results from the glycosaminoglycans (sGAG) in the cartilage. Consequently, ioxaglate accumulation in the cartilage is inversely related to the sGAG content. To obtain this, all joints and the different body parts were, before imaging, immersed for $30 \mathrm{~min}$ in a solution of Hexabrix ${ }^{\circledR} 320$ diluted $4 / 5$ in PBS, then wrapped in parafilm and stably positioned in the nanoCT system. The mouse newborn was, without any sample preparation, immersed overnight in a solution of Hexabrix ${ }^{\circledR} 320$ diluted 8/9 in PBS, then positioned in an Eppendorf tube that was sealed with parafilm, and stably positioned in the nanoCT system for imaging.

\section{D visualisation and image analysis}

For $3 \mathrm{D}$ visualisation of the bone and cartilage of the mouse newborn and the femora and/or tibiae, Mimics (Materialise, Haasrode, Belgium) was applied. To quantify the thickness of the non-calcified and calcified cartilage layers, the cartilage region was selected using CTAn software (Bruker micro-CT, Kontich, Belgium) by manually - but consistently - drawing a region of interest (ROI) in every $20^{\text {th }}$ cross-section throughout the whole tibial plateau. Delineating the total cartilage area (i.e. both non-calcified and calcified) was done by readers, blinded for the experimental conditions, and was based on (i) the difference in grey-scale from the surroundings (i.e. liquid) for the non-calcified cartilage, and (ii) the difference in structure compared to the subchondral bone and noncalcified cartilage for the calcified cartilage (i.e. having a porous structure). The ROI was interpolated automatically for the intermediate cross-sections. By selecting a manually chosen global threshold, the non-calcified and calcified cartilage were segmented and analysed in 3D by calculating the volume, thickness distribution and average thickness. The latter two were calculated using a method defined by Hildebrand and Ruëgsegger (1997). 


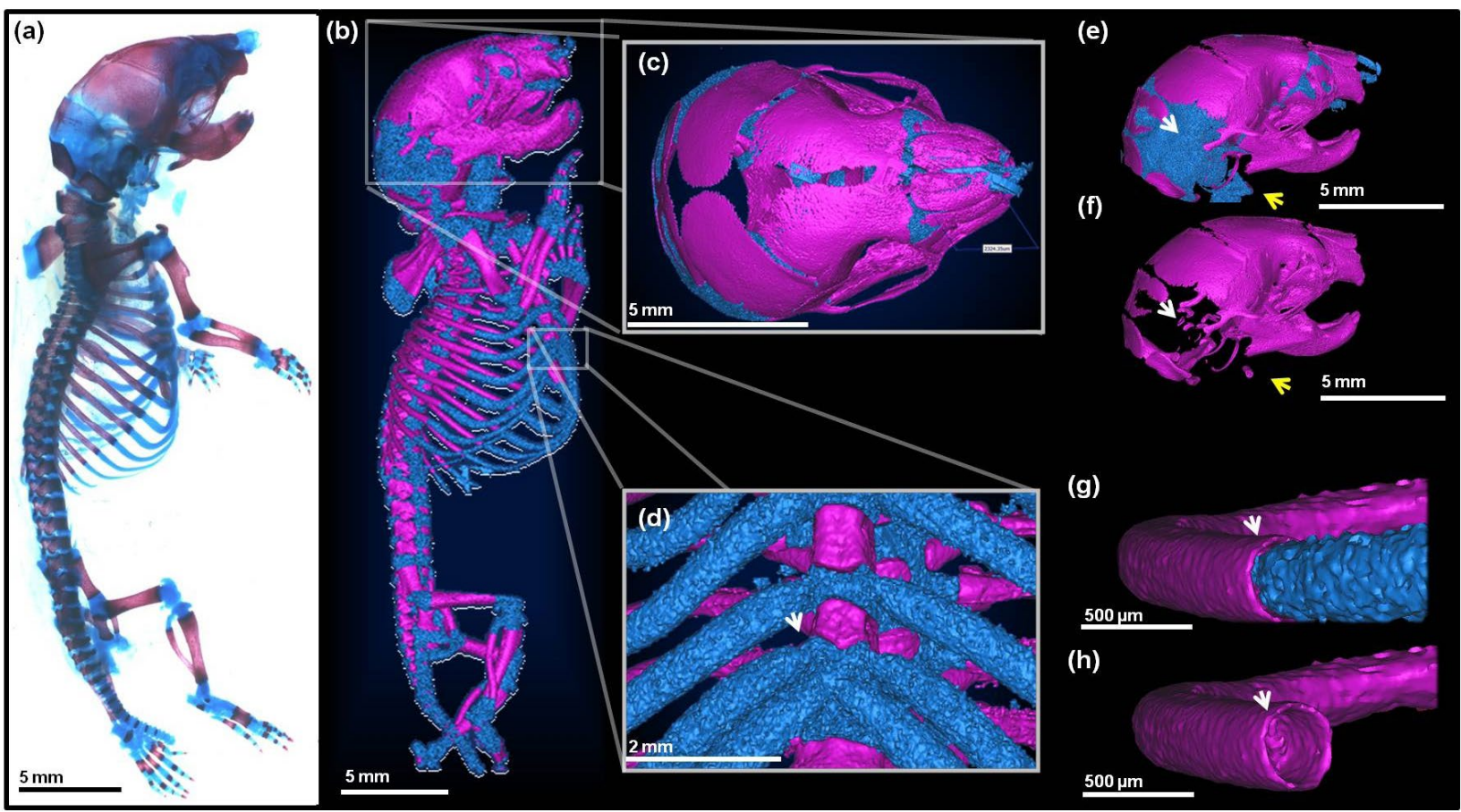

Fig. 1. (a) Whole mount skeletal stain of a mouse newborn (E18.5) and its (b) 3D reconstruction based on the CEnanoCT dataset. In both, the pink colour represents bony and mineralised tissue and blue reveals the cartilage. (c) A zoom of the skull of the newborn showing the bone and mineralised tissue. (d) A zoom of (b) showing the sternum at the level where the last true ribs fuse (white arrow). (e) The skull of the newborn showing cartilaginous, bone and mineralised tissue, where, like the whole mount staining, the internal parts are not visible, and (f) the same image as (e), where the cartilage tissue has been digitally omitted revealing the ear bones (white arrow) and hyoid bone (yellow arrow). (g) The transition point between cartilage and bone in the chondro-osseous junction of a mouse rib (white arrow), and (h) the same image without cartilage tissue, showing the "outside-in" replacement of cartilage by bony tissue.

\section{Image validation}

The obtained CE-nanoCT images were compared to the following standard staining procedures:

Whole mount skeletal staining

Alcian blue/alizarin red staining of cartilage and bone was performed on a cleared newborn (E18.5) as described by Nagy et al. (2003).

Histology

After nanoCT imaging, the tissues were processed for histology. They were fixed in $10 \%$ formalin, if appropriate - decalcified in $4 \%$ formic acid, and embedded in paraffin. For the joints, sections of $5 \mu \mathrm{m}$ were made every $60 \mu \mathrm{m}$ and stained with Safranin-O and counterstained with Fast Green, resulting in images where bone stains light blue, calcified cartilage stains light red and non-calcified hyaline cartilage stains dark red.

\section{Statistical analysis}

Statistical analysis was performed using a statistical software add-in for Microsoft ${ }^{\circledR}$ Excel $^{\circledR}$ for Windows, namely Analyse-it version 2.26 Excel 12+. An unpaired Student's $t$-test was used to calculate the significance level between the Sham and DMM group for the average thickness of calcified and non-calcified cartilage. Data was significantly different for $p$-values less than 0.05 .

\section{Results}

\section{CE-nanoCT allows 3D visualisation and digital dissection of a mouse newborn}

As a first important and original application, we show that a CE-nanoCT scan of a newborn mouse (Fig. 1b) can visualise bone and cartilage much like the traditional whole mount Alcian Blue-Alizarin Red skeletal stain (Fig. 1a), i.e. the red-pink colour represents bone and mineralised tissue and blue indicates cartilage. The high-resolution digital reconstituted 3D image obtained by CE-nanoCT allows easy manipulation and 'digital dissection' to evaluate and quantify size, structure and composition of internal, microscale structures, e.g. skull (Fig. 1c) or sternum (Fig. 1d). The high spatial resolution even allows one to zoom in on small internal tissues composed of bony or cartilaginous material or a combination thereof. This is exemplified in Figs. 1e-h, where (i) the inner ear bones (white arrow in Figs. 1e and 1f) become visible after digitally masking the cartilaginous tissue, and (ii) typical chondro-osseous junctions are shown, such as the hyoid bone (yellow arrow in Figs. 1e and 1f) and the transition of the bone and cartilaginous part of ribs, revealing the gradual "outsidein" growth and differentiation of cartilage into bone (Figs. $\lg$ and $1 \mathrm{~h})$. 


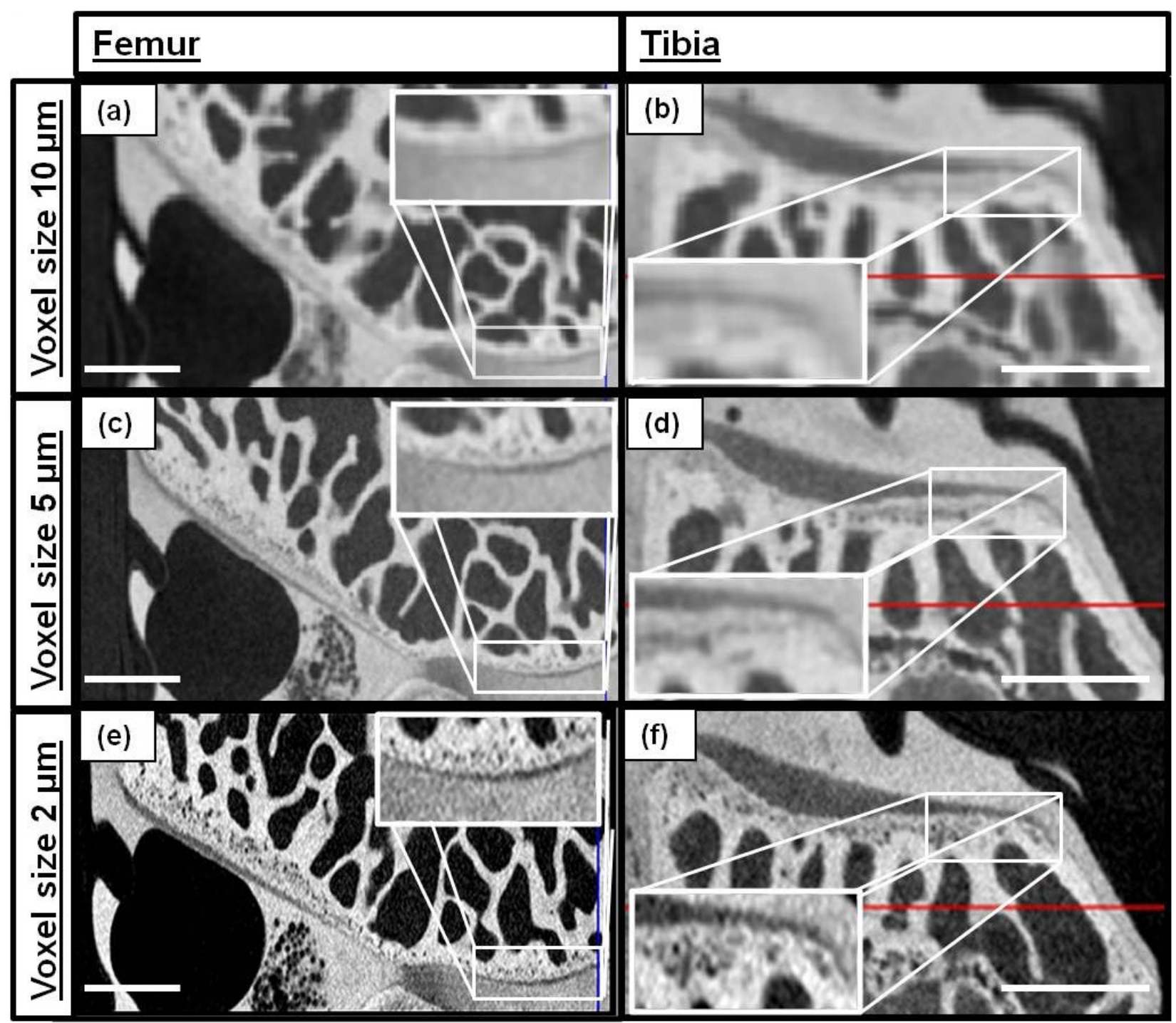

Fig. 2. A representative coronal CE-nanoCT section through a (a, c, e) femoral condyle and $(\mathbf{b}, \mathbf{d}, \mathbf{f})$ tibial plateau scanned with an isotropic voxel size of (a, b) $10 \mu \mathrm{m}, \mathbf{( c ,}, \mathbf{d}) 5 \mu \mathrm{m}$ and (e, f) $2 \mu \mathrm{m}$. Within each image, a magnification of a part of the chrondro-osseous junction is shown. The non-calcified cartilage appears in dark grey and the light grey porous layer is the calcified cartilage. The white to light grey dense structure is the subchondral bone. Scale bar $=250 \mu \mathrm{m}$.

CE-nanoCT allows discrimination between bone, calcified articular cartilage and non-calcified articular cartilage in 3D

Fig. 2 shows typical coronal CE-nanoCT sections through a femoral condyle and a tibial plateau that were scanned with a varying isotropic voxel size, ranging from those that have been reported using EPIC-CT (Kotwal et al., 2012; Liang et al., 2011; Palmer et al., 2006; Piscaer et al., 2008; Xie et al., 2010; Xie et al., 2009) to the highest obtainable with CE-nanoCT for the given sample size. The non-calcified hyaline cartilage is shown in dark grey, the light grey porous layer is the underlying calcified cartilage, and the light grey dense structure is the subchondral bone. In the $10 \mu \mathrm{m}$ voxel size images of the femoral condyle and the tibial plateau (Figs. 2a and 2b), no distinction was possible between calcified cartilage and subchondral bone, nor was it possible to clearly delineate the non-calcified cartilage. Thus, quantification of the thickness of both layers was not feasible. In the $5 \mu \mathrm{m}$ voxel size images (Figs. $2 \mathrm{c}$ and $2 \mathrm{~d}$ ), one could only distinguish the non-calcified cartilage in areas where the thickness of the layer is larger than $15 \mu \mathrm{m}$. It can be appreciated that only the highest spatial image resolution (i.e. isotropic voxel size of $2 \mu \mathrm{m}$, Figs. 2e and 2f) can clearly visualise and discriminate the transition between the non-calcified and the calcified cartilage as well as the chondro-osseous junction, thus enabling quantification of the properties of these layers.

To confirm the sublineage-specificity of CE-nanoCT, femoral condyles treated with papain, specifically removing the non-calcified cartilage layer (Hughes et al., 2005), were compared to untreated femoral condyles by both histological sectioning (Figs. 3a and 3d) and CE-nanoCT (Figs. 3b, 3c, 3e and 3f). After papain treatment, the porous calcified cartilage layer was still clearly visible on both the histological and CE-nanoCT images (highlighted in Fig. 3f), whereas the dark-gray non-calcified layer had disappeared. This thus confirmed the unprecedented discriminative power of CE-nanoCT. 


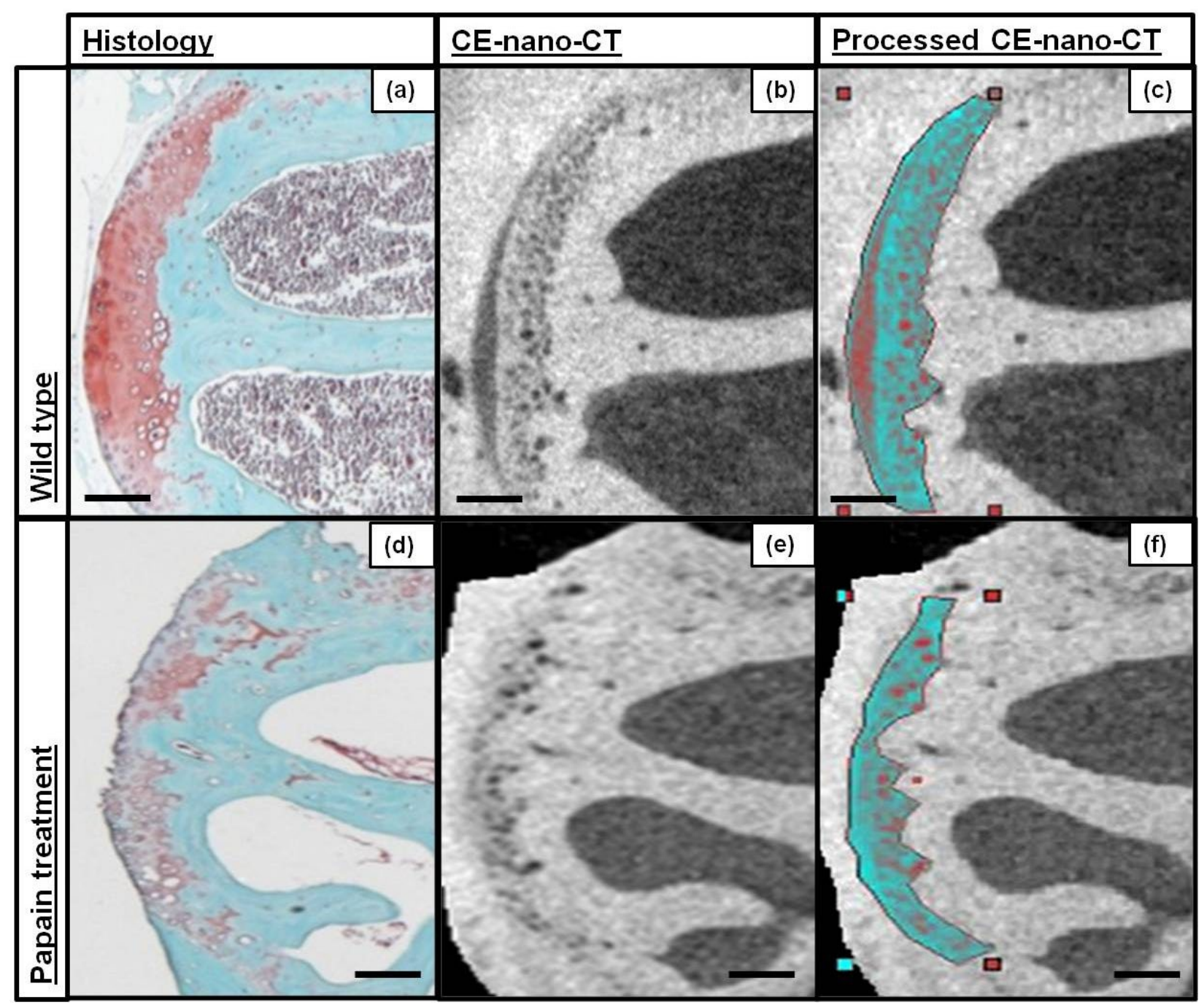

Fig. 3. (a, d) A representative coronal histological section (Safranin-O/Fast green staining) through the femoral condyles, $\mathbf{( b ,} \mathbf{e})$ the corresponding CE-nanoCT sections, and $(\mathbf{c}, \mathbf{f})$ the same CE-nanoCT section with the traced regions of interest (i.e. total (non-calcified and calcified) hyaline cartilage) of (a-c) an untreated mouse condyle and (d-f) a mouse condyle treated with papain to specifically remove the non-calcified cartilage layer, but preserve the calcified cartilage layer. Scale bars $=200 \mu \mathrm{m}$.

\section{CE-nanoCT allows 3D visualisation and} quantification of the cartilage structure and functionality in an in vivo model for osteoarthritis

The broad applicability of CE-nanoCT was also validated on adult mouse tibia after in vivo meniscus destabilisation, which serves as a model for OA (Glasson et al., 2007). The model is characterised by mild inflammation and cartilage lesions of various degrees of severities. Figs. $4 a$ and $4 b$ both clearly visualise a decrease in sGAG content in the non-calcified cartilage layer, whereas in Figs. $4 c$ and $4 d$ chondral lesions can be depicted. CE-nanoCT is thus able to identify and distinguish between an alteration of cartilage composition and cartilage lesions.

Quantification of the non-calcified and calcified hyaline cartilage volume, averaged over the total tibial plateau ( $n=4$ per group), showed local defects with a volume of $0.045 \pm 0.018 \mathrm{~mm}^{3}$ (i.e. $43.8 \% \pm 18.9 \%$ loss in noncalcified cartilage volume). The loss in non-calcified cartilage volume ranged from $24.4 \%$ to $64.2 \%$, which is similar to the range mentioned by Glasson et al. (2007).
Statistical analysis $(n=4)$ revealed a significant decrease in average thickness of the non-calcified cartilage, but leaving the underlying calcified cartilage mostly unaffected (Fig. 5a). This was confirmed in Figs. $5 \mathrm{~b}$ and 5c, which represent a 3D model based on CE-nanoCT images of such a DMM mouse (i.e. the mouse with the largest lesion within the DMM group: up to $0.061 \mathrm{~mm}^{3}$ or $64 \%$ loss in non-calcified cartilage volume to exemplify how severe the lesions may be) and a non-treated littermate, and in the averaged thickness distribution plots $(n=4)$ of non-calcified and calcified cartilage (Figs. 5d and 5e respectively). It needs to be mentioned that, although the angle at which the $3 \mathrm{D}$ image in Fig. 5b is shown, the impression might be given that the cartilage area on the medial side was much larger than on the lateral side - this was not the case. The mouse that had undergone an in vivo meniscus destabilisation (Fig. 5c) displayed, in 3D, a severe lesion within the noncalcified cartilage on the medial side, which is in line with the literature on the pathology in this model (Glasson et al., 2007), but so far has lacked quantification. 


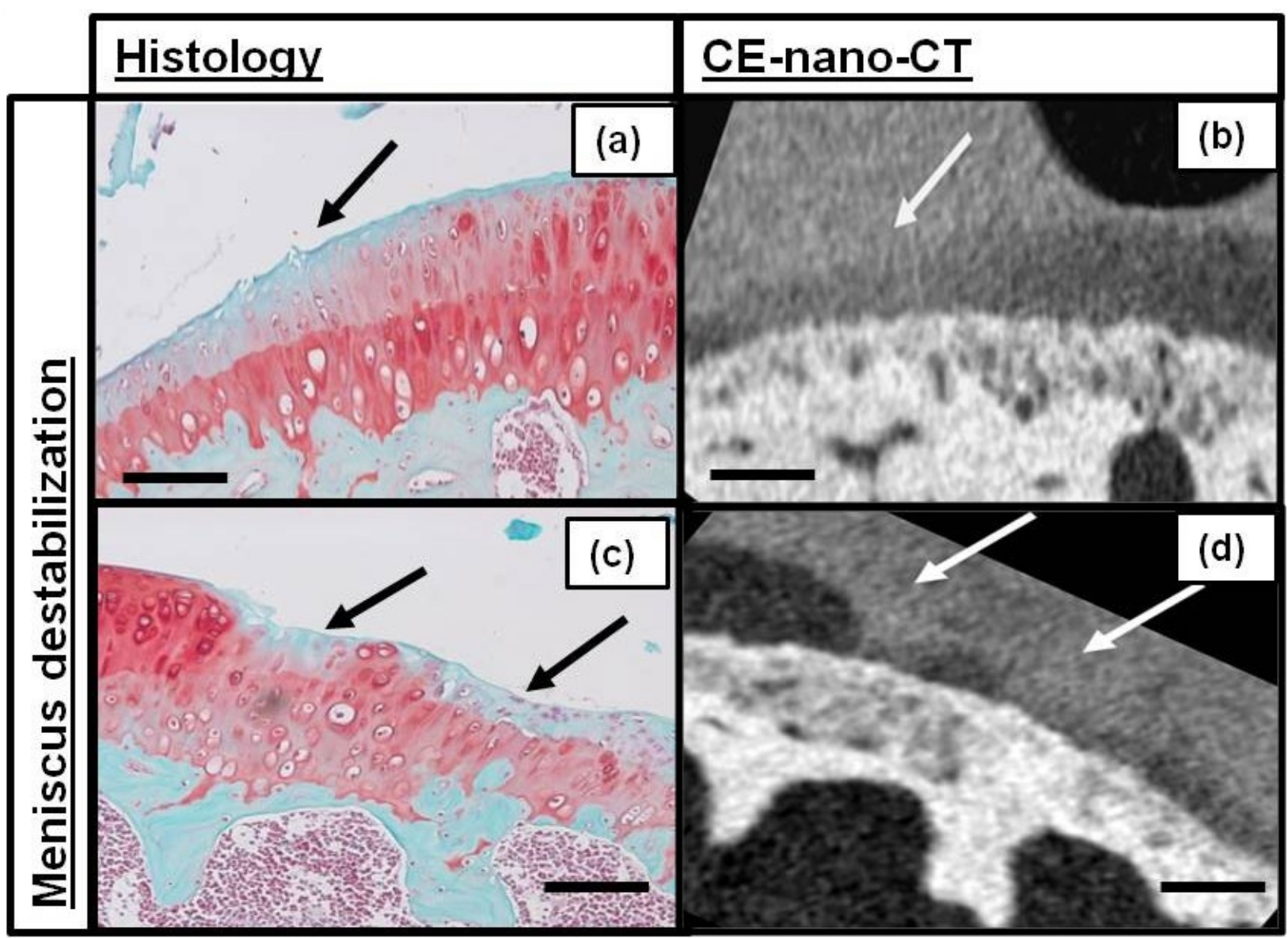

Fig. 4. (a, c) A representative histological section and $(\mathbf{b}, \mathbf{d})$ the corresponding CE-nanoCT sections through the tibial plateau of mice that had undergone in vivo meniscus destabilisation, resulting in a condyle with (a, b) a decrease in sGAG content in the non-calcified cartilage layer and $(\mathbf{c}, \mathbf{d})$ two lesions. Scale bars $=200 \mu \mathrm{m}$.

\section{CE-nanoCT allows visualising in 3D different forms} of cartilage and tissue combinations

We validated CE-nanoCT on different forms of cartilage within their surrounding tissue by comparing them to histological staining. Fig. 6 shows a typical CE-nanoCT section through a mouse trachea (Fig. 6b) and patella (Fig. 6d), with a corresponding histological section (Figs. $6 \mathrm{a}$ and $6 \mathrm{c}$, respectively). The digital CE-nanoCT section of the mouse trachea (Fig. 6b) exemplifies infiltration of Hexabrix ${ }^{\circledR}$ into the pericellular matrix of the chondrocytes, which allows visualisation of the lacunae. The CE-nanoCT image of the mouse patella (Fig. 6d) revealed clearly the articular cartilage that slides over the femoral cartilage (yellow arrow). On the opposite side (white arrow), a highly structured and oriented calcified cartilaginous layer is apparent, which serves as the connection to the patellar tendon. Besides, tendons, which also contain sGAG, and muscle show up in the same grey-scale as the non-calcified cartilage (yellow arrow, Fig. 6f; muscle is the feather-like structure in the paw of the mouse, white arrow) although it appears negative following SafraninO staining (Fig. 6e, black arrow).

\section{Discussion}

To date, magnetic resonance imaging is, due to its high sensitivity for discriminating soft tissues, the standard technique for the visualisation and quantification of cartilage morphology and functionality (Burstein et al., 2001; Kuperman, 2000). For bone and mineralised tissue, $\mathrm{X}$-ray computed tomography $(\mathrm{CT})$ is the imaging technique of choice (Cartmell et al., 2004; Hagenmueller et al., 2007; Porter et al., 2007). However, for lab-based research, where small animal models are typically used to evaluate the efficacy of different therapies for bone and cartilage repair or to evaluate cartilage and bone during foetal and postnatal development, both techniques suffer from shortcomings in the offered spatial image and/or contrast resolution. In addition, the use of two different techniques to visualise adjacent tissues does not allow for a detailed analysis of the structure, function and pathophysiology of the chondro-osseous junction, which is for example key in understanding the pathogenesis of joint diseases such as OA (Glasson et al., 2007).

Micro-CT has been reported frequently for imaging and assessing ex vivo the effect of different types of arthritis on the bone structure (Buttgereit et al., 2009; Herrak et al., 2004), surface roughness (Seeuws et al., 2010) and density (Healy et al., 2006). Although this technique allows investigation of the effect of arthritis on the underlying bone, nothing can be concluded concerning cartilage structure and functionality. Additionally, because of its limited spatial resolution and field of view, only selected parts of the animal (i.e. paw or knee joints) are assessed. Micro-CT has also been used to look at skeletal 

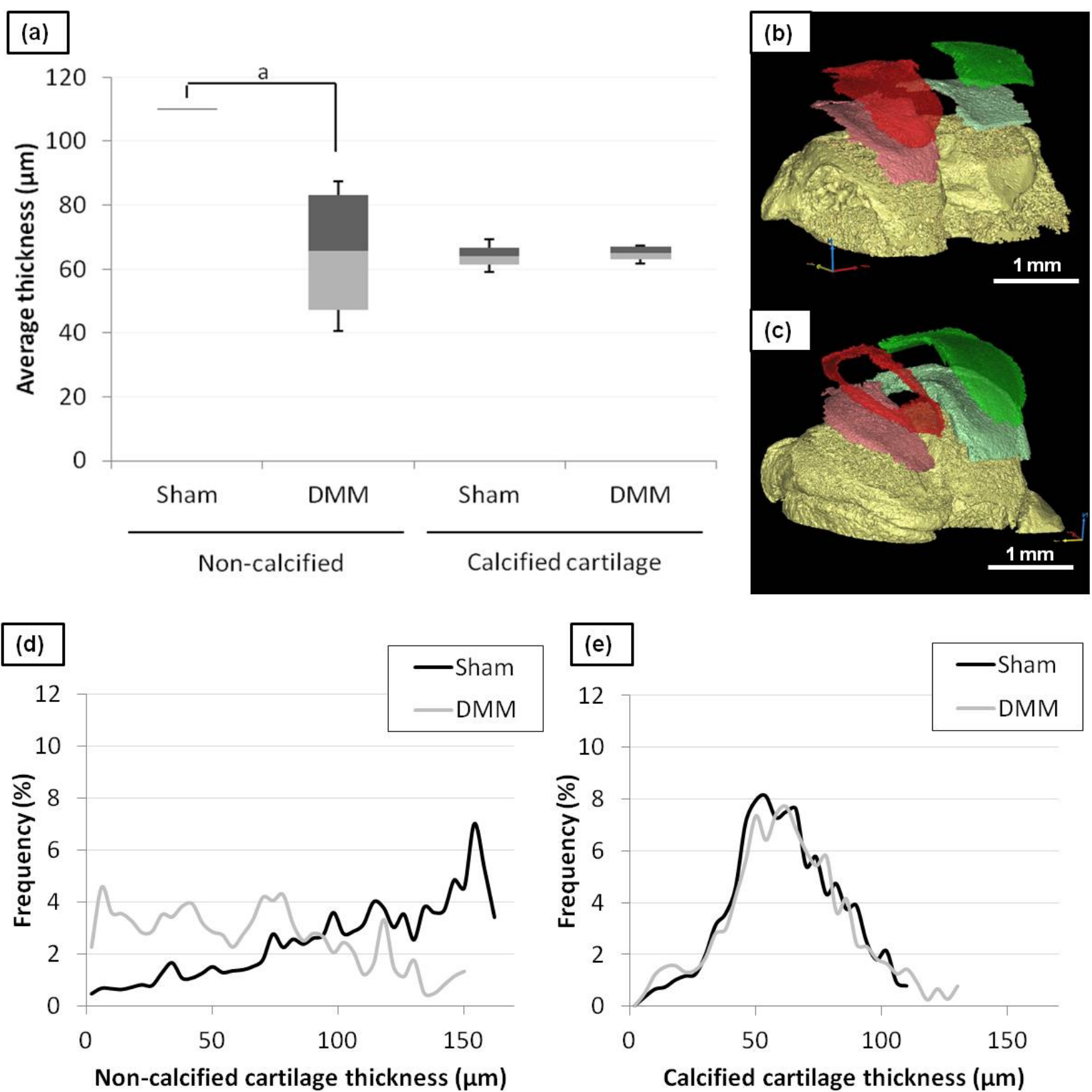

Fig. 5. (a) Box plots of the average thickness of non-calcified and calcified cartilage on the medial tibial plateau of wild type mice (Sham; $n=4$ ) and mice with a destabilised meniscus (DMM; $n=4$ ) [ $\left.{ }^{\mathrm{a}} p=0.03\right]$. A 3D assembly of a tibia of (b) an untreated mouse and (c) a mouse that had undergone an in vivo meniscus destabilisation. The non-calcified cartilage layer on the lateral side of the tibia is bright green and transparent, and the calcified cartilage layer in pale green. On the medial side, the non-calcified cartilage layer is bright red and transparent, and the calcified cartilage layer is pale red. Bone is shown in yellow. The averaged thickness distribution plot of the (d) non-calcified and (e) calcified cartilage on the medial tibial plateau of an untreated mouse (Sham; $n=4$ ) and mouse with a destabilised meniscus (DMM; $n=4)$.

development (Liakhovitskaia et al., 2010). Although fully in $3 \mathrm{D}$, only the mineralised parts can be imaged and analysed.

As a solution, the combination of Hexabrix ${ }^{\circledR}$, a radio-opaque negatively charged ionic iodinated dimer, with micro-CT (i.e. EPIC-CT) has been employed for simultaneously imaging bone and cartilage in 3D (Kotwal et al., 2012; Liang et al., 2011; Palmer et al., 2006; Piscaer et al., 2008; Xie et al., 2010; Xie et al., 2009). For example, Piscaer et al. (2008) applied Hexabrix ${ }^{\circledR}$ in vivo to visualise and quantify cartilage degeneration in rats. Palmer et al.
(2006) and Xie et al. (2010) performed similar experiments by means of ex vivo micro-CT. However, EPIC-CTs spatial image resolution is restricted, and therefore is limited in its use for assessment of the mouse model, where the thickness of the hyaline cartilage can be down to some tens of microns (Kotwal et al., 2012). Indeed, in this study we have shown that earlier reported spatial image resolutions (Kotwal et al., 2012; Liang et al., 2011; Palmer et al., 2006; Piscaer et al., 2008; Xie et al., 2010; Xie et al., 2009) could not clearly discriminate calcified cartilage from subchondral bone. Hence, the total cartilage, which consists 


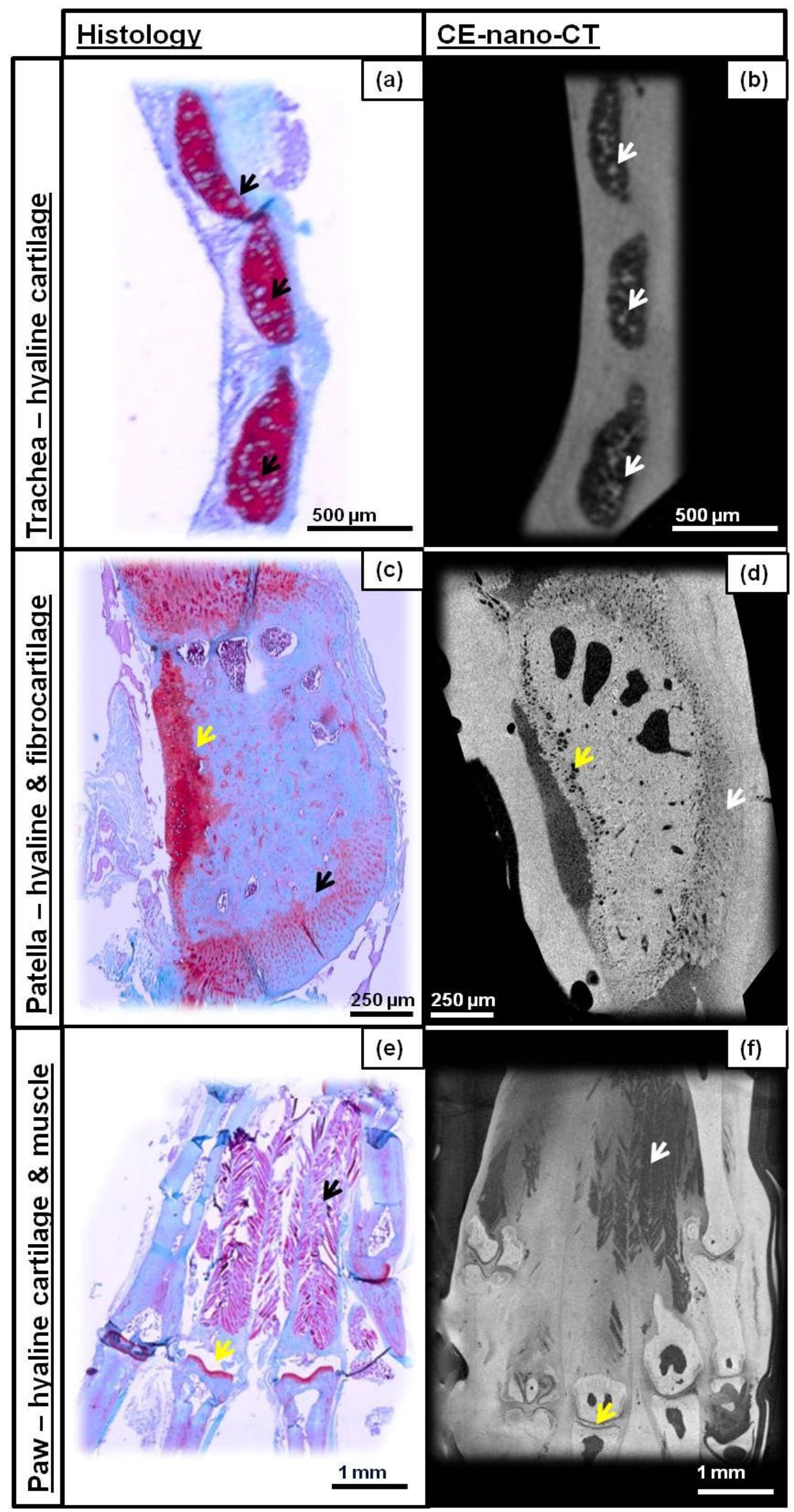

Fig. 6. A coronal histological section (SafO/Fast green staining) and a corresponding CE-nanoCT section through the mouse (a, b) trachea, showing lacunae (arrows), (c, d) patella with the articular cartilage (yellow arrow) and on the opposite side (black/white arrow) a highly structured and oriented calcified cartilaginous layer, and (e, f) paw, where, apart from the articular cartilage (yellow arrow), the muscle (black/white arrow) may be visualised. 
of the calcified and non-calcified zone, was thus likely underestimated in these studies. We here have introduced an improved non-invasive imaging methodology compared to EPIC-CT, i.e. CE-nanoCT, which allows simultaneous imaging of cartilage and bone at a novel contrast and spatial resolution, and allows discriminating between calcified cartilage and subchondral bone; hence all can be visualised and quantified in one image dataset.

Figs. 5d and 5e present averaged distribution plots for a Sham operated group and animals that had undergone medial meniscus destabilisation (DMM). For the first group, where the average non-calcified cartilage thickness is well above $15 \mu \mathrm{m}$ (Fig. 5a), only $2.4 \pm 0.3 \%$ of the non-calcified cartilage thickness is below $15 \mu \mathrm{m}$. For this group, the $5 \mu \mathrm{m}$ voxel size images might be sufficient for $3 \mathrm{D}$ quantification. However, when severe lesions occur, such as in the case of the DMM group, about 14.5 $\pm 7.7 \%$ of the non-calcified cartilage thickness is below $15 \mu \mathrm{m}$, and about $10.5 \pm 5.6 \%$ of the thickness is even below $10 \mu \mathrm{m}$. In these regions, the limited spatial image resolution of EPIC-CT will not suffice to capture important differences, and would exclude the analysis of the full architecture of calcified and non-calcified cartilage and the underlying bone, while the highest attainable resolution of CE-nanoCT for these samples allowed quantification of the thickness and quality of these different layers. This may for example be of importance to understand the transport between the subchondral bone and non-calcified cartilage, and the early effects of OA (Botter et al., 2008), as well as other diseases such as craniosynostosis or achondroplasia. An additional argument for the requirement of the high contrast and spatial image resolution of CE-nanoCT is that $\mathrm{OA}$ is a disease that affects the whole joint and is characterised by articular cartilage destruction along with changes occurring in other joint components, including bone, menisci, synovium, ligaments, capsule and muscles. All these tissues influence each other and there is still discussion on what is the primary cause of OA. Some say that the cause of OA might be an inflammation of the underlying bone. To assess all different tissues involved, it is important to be able to discriminate them. Another argument may come from the study of Ea et al. (2011) on calcification in the pathology of OA, whereby calcification apparently precedes the appearance of cartilage lesions. If one wishes to determine such events, high resolution and contrast imaging that is able to discriminate calcified nodules in non-calcified cartilage, such as CE-nanoCT, is required.

In this study, the thickness of the non-calcified and calcified cartilage layers was quantified in $3 \mathrm{D}$ by manually selecting an ROI delineating the total cartilage area (i.e. non-calcified and calcified) in every $20^{\text {th }}$ cross-section over the full tibial plateau. The non-calcified cartilage was separated from the background (liquid) based on a difference in grey-scale. As can be seen in Fig. 4, in regions where the sGAG content was low and hence Hexabrix ${ }^{\circledR}$ had partially penetrated the non-calcified cartilage, it was difficult to distinguish between background and noncalcified cartilage in an automated way. Hence, we have chosen to manually and consistently trace the non-calcified cartilage in the cross-sections. The calcified cartilage, being porous and thus distinctly different in structure from the surrounding tissues (i.e. subchondral bone and noncalcified cartilage), allows a much more straightforward delineation.

We showed that CE-nanoCT allows for the study of the architecture of various types of cartilaginous tissue in a small rodent model. By testing this technique on various tissues and on mice of different ages, we showed that the technology can be implemented widely and thus may be used to follow normal and pathological developmental processes in mutant animals, and to address various pathologies of bone and cartilaginous tissue as well as processes of aging. Indeed, the unprecedented 3D skeletal reconstruction of a full mouse pup shown in this study indicates that $\mathrm{CE}$-nanoCT datasets allow imaging both the bone and cartilage of a mouse embryo or newborn in one single scan. It enables an easier, faster and more detailed 3D quantitative assessment (i.e. sGAG content, bone density,...) of the form, size or volume of bone and cartilaginous tissue during normal and pathological processes, or malformations as a consequence of aberrant skeletal development, as compared to state-of-the-art skeletal staining. Moreover, internal features can be visualised and isolated by digital extraction eliminating the need for extensive sample dissection.

Although EPIC-CT has proven its validity to assess preclinical efficacy of novel therapies for bone and cartilage repair, Modified Mankin scoring of histological sections is still mostly used as the standard evaluation technique due to its widespread adoption, ease of use and its similarity to scoring systems used for OA in humans (Glasson et al., 2010). This method focuses on the structural changes observed, such as lesions or fibrillations and losses in proteoglycan staining. Immunohistochemistry has a high discriminative power but is end stage, destructive, labour intensive, and only provides $2 \mathrm{D}$ data. Because of the high discriminative power of CE-nanoCT, resulting in images comparable to the Safranin-O/Fast green-stained histological sections typically used for the Modified Mankin scoring, we can speculate that the CE-nanoCT images can be used with similar accuracy as histological sections for Modified Mankin scoring (ongoing work). Moreover, CE-nanoCT allows scoring in a $3 \mathrm{D}$ setting, eliminating the 2D character of the traditional histological methodology, which potentially enables capturing smaller alterations in cartilage structure. It is tempting to speculate that, given the unprecedented discriminative and quantitative power of CE-nanoCT at subtissue level, it should be feasible to implement the technique to small animal models for cartilage diseases other than OA, where CE-nanoCT may reveal so far overlooked therapeutic efficacies.

The combination of a contrast agent with the high spatial resolution of nanoCT has some limitations. Hexabrix $^{\circledR}$ is a negatively charged ionic iodinated dimer, and hence will be excluded from tissues that are negatively charged. Therefore, not only cartilage but also tendons, which also contain sGAG, and muscle will show up in the $\mathrm{CE}$-nanoCT images having the same grey-scale as the non-calcified cartilage. This is exemplified in Fig. 6 for the muscle (feather-like structure) in the paw of the 
mouse, which is devoid of sGAG as it does not stain pink upon Safranin/O staining in the corresponding histological section. In particular cases, this may generate some background, but may - in addition to the chrondro-osseous interactions - allow visualisation of the musculo-skeletal interaction.

Moreover, the high X-ray doses needed to obtain the required high spatial image and contrast resolution with nanoCT do currently not allow in vivo imaging of hyaline cartilage subtissues. In addition, Turunen et al. (2011) have shown that Hexabrix ${ }^{\circledR}$ may reduce the stiffness of the articular cartilage, which is further hypothesised to lead to overload-induced cell death. This thus excludes the use of the said technique, and in particular the use of Hexabrix ${ }^{\circledR}$ as a contrast agent, for in vivo time-lapsed imaging of cartilage.

\section{Conclusions}

We have implemented a significantly enhanced noninvasive 3D imaging methodology, i.e. CE-nanoCT, that allows simultaneous imaging of cartilage and bone at a novel contrast and spatial resolution. By combining the use of an anionic contrast agent (i.e. Hexabrix ${ }^{\circledR}$ ) with the high resolution of nanoCT, CE-nanoCT can reveal differences in structure and composition between calcified cartilage, non-calcified cartilage and the underlying bone of small animal models. By testing this technique on various tissues in different body parts and on mice of different ages, we showed that the technology can be implemented widely. Because of its ability to provide faster, improved and more quantitative data on cartilage structure and composition, CE-nanoCT has demonstrated potential as an enabling technology for preclinical small animal studies especially addressing cartilage-related pathologies and therapeutic interventions. In addition, the 3D models at the same time allow studying morphology and spatial distribution of the cartilage.

\section{Acknowledgements}

This study was financed by the Agency for Innovation by Science and Technology in Flanders (IWT/OZM/090655). We are very grateful to G. Vanderlinden, J. Op De Beeck, J. Neys and M. Hemelaers for excellent technical assistance. We also thank Dr. P. Tylzanowski for performing the skeletal staining of the mouse newborn. This work is part of Prometheus, the Leuven Research \& Development Division of Skeletal Tissue Engineering of the KU Leuven: www.kuleuven.be/prometheus. We wish to confirm that there are no known conflicts of interest associated with this publication and there has been no significant financial support for this work that could have influenced its outcome.

\section{References}

Botter SM, van Osch GJ, Waarsing JH, van der Linden JC, Verhaar JA, Pols HA, van Leeuwen JP, Weinans H (2008) Cartilage damage pattern in relation to subchondral plate thickness in a collagenase-induced model of osteoarthritis. Osteoarthritis Cartilage 16: 506-514.

Burstein D, Velyvis J, Scott KT, Stock KW, Kim YJ, Jaramillo D, Boutin RD, Gray ML (2001) Protocol issues for delayed Gd(DTPA)(2-)-enhanced MRI (dGEMRIC) for clinical evaluation of articular cartilage. Magn Reson Med 45: 36-41.

Buttgereit F, Zhou H, Kalak R, Gaber T, Spies CM, Huscher D, Straub RH, Modzelewski J, Dunstan CR, Seibel MJ (2009) Transgenic disruption of glucocorticoid signaling in mature osteoblasts and osteocytes attenuates $\mathrm{K} / \mathrm{BxN}$ mouse serum-induced arthritis in vivo. Arthritis Rheum 60: 1998-2007.

Cartmell S, Huynh K, Lin A, Nagaraja S, Guldberg R (2004) Quantitative microcomputed tomography analysis of mineralization within three-dimensional scaffolds in vitro. J Biomed Mater Res A 69A: 97-104.

Ea HK, Nguyen C, Bazin D, Bianchi A, Guicheux J, Reboul P, Daudon M, Liote F (2011) Articular cartilage calcification in osteoarthritis: insights into crystal-induced stress. Arthritis Rheum 63: 10-18.

Glasson SS, Blanchet TJ, Morris EA(2007) The surgical destabilization of the medial meniscus (DMM) model of osteoarthritis in the $129 / \mathrm{SvEv}$ mouse. Osteoarthritis Cartilage 15: 1061-1069.

Glasson SS, Chambers MG, Van Den Berg WB, Little CB (2010) The OARSI histopathology initiative - recommendations for histological assessments of osteoarthritis in the mouse. Osteoarthritis Cartilage 18 Suppl 3: S17-23.

Hagenmueller H, Hofmann S, Kohler T, Merkle HP, Kaplan DL, Vunjak-Novakovic G, Mueller R, Meinel L (2007) Non-invasive time-lapsed monitoring and quantification of engineered bone-like tissue. Ann Biomed Eng 35: 1657-1667.

Healy AM, Izmailova E, Fitzgerald M, Walker R, Hattersley M, Silva M, Siebert E, Terkelsen J, Picarella D, Pickard MD, LeClair B, Chandra S, Jaffee B (2006) PKCtheta-deficient mice are protected from Th1-dependent antigen-induced arthritis. J Immunol 177: 1886-1893.

Herrak P, Gortz B, Hayer S, Redlich K, Reiter E, Gasser J, Bergmeister H, Kollias G, Smolen JS, Schett G (2004) Zoledronic acid protects against local and systemic bone loss in tumor necrosis factor-mediated arthritis. Arthritis Rheum 50: 2327-2337.

Hildebrand T, Ruegsegger P (1997) A new method for the model-independent assessment of thickness in threedimensional images. J Microsc 185: 67-75.

Hughes LC, Archer CW, ap Gwynn I (2005) The ultrastructure of mouse articular cartilage: collagen orientation and implications for tissue functionality. A polarised light and scanning electron microscope study and review. Eur Cell Mater 9: 68-84.

Huisken J, Swoger J, Del Bene F, Wittbrodt J, Stelzer EH (2004) Optical sectioning deep inside live embryos by selective plane illumination microscopy. Science $\mathbf{3 0 5}$ : 1007-1009.

Kotwal N, Li J, Sandy J, Plaas A, Sumner DR (2012) Initial application of EPIC-muCT to assess mouse articular cartilage morphology and composition: effects of aging and treadmill running. Osteoarthritis Cartilage 20: 887-895. 
Kuperman V (2000) Magnetic Resonance Imaging: Physical Principles And Applications, Chapter 1. Academic Press, San Diego.

Liakhovitskaia A, Lana-Elola E, Stamateris E, Rice DP, van't Hof RJ, Medvinsky A (2010) The essential requirement for Runx1 in the development of the sternum. Dev Biol 340: 539-546.

Liang G, Vanhouten J, Macica CM (2011) An atypical degenerative osteoarthropathy in Hyp mice is characterized by a loss in the mineralized zone of articular cartilage. Calcif Tissue Int 89: 151-162.

Lyons SK (2005) Advances in imaging mouse tumour models in vivo. J Pathol 205: 194-205.

Maye P, Fu Y, Butler DL, Chokalingam K, Liu Y, Floret J, Stover ML, Wenstrup R, Jiang X, Gooch C, Rowe D (2011) Generation and characterization of Col10a1mcherry reporter mice. Genesis 49: 410-418.

Nagy A, Gertsenstein M, Vintersten K, Behringer RR (2003) Manipulating the Mouse Embryo: A Laboratory Manual, Chapter 16. Cold Spring Harbor Press, New York.

Palmer AW, Guldberg RE, Levenston ME (2006) Analysis of cartilage matrix fixed charge density and three-dimensional morphology via contrast-enhanced microcomputed tomography. Proc Natl Acad Sci USA 103: 19255-19260.

Piscaer TM, Waarsing JH, Kops N, Pavljasevic P, Verhaar JAN, van Osch GJVM, Weinans H (2008) In vivo imaging of cartilage degeneration using [mu]CTarthrography. Osteoarthritis Cartilage 16: 1011-1017.

Porter BD, Lin ASP, Peister A, Hutmacher D, Guldberg RE (2007) Noninvasive image analysis of 3D construct mineralization in a perfusion bioreactor. Biomaterials 28: 2525-2533.

Sasov A (2004) X-ray nanotomography. In: Bonse U (ed) Developments in X-Ray Tomography IV. Proc SPIE 5535: 201-211.

Seeuws S, Jacques P, Van Praet J, Drennan M, Coudenys J, Decruy T, Deschepper E, Lepescheux L, Pujuguet P, Oste L, Vandeghinste N, Brys R, Verbruggen G, Elewaut D (2010) A multiparameter approach to monitor disease activity in collagen-induced arthritis. Arthritis Res Ther 12: R160.

Sharpe J, Ahlgren U, Perry P, Hill B, Ross A, HecksherSorensen J, Baldock R, Davidson D (2002) Optical projection tomography as a tool for 3D microscopy and gene expression studies. Science 296: 541-545.

Turunen MJ, Toyras J, Lammi MJ, Jurvelin JS, Korhonen RK (2011) Hyperosmolaric contrast agents in cartilage tomography may expose cartilage to overloadinduced cell death. J Biomech 45: 497-503.

Xie L, Lin ASP, Levenston ME, Guldberg RE (2009) Quantitative assessment of articular cartilage morphology via EPIC-mu CT. Osteoarthritis Cartilage 17: 313-320.

Xie L, Lin ASP, Guldberg RE, Levenston ME (2010) Nondestructive assessment of sGAG content and distribution in normal and degraded rat articular cartilage via EPIC-mu CT. Osteoarthritis Cartilage 18: 65-72.

\section{Discussion with Reviewer}

Reviewer II: The main overall issue I have with this work is: Do the quantitative measurements you show in the paper actually demonstrate the high resolution potential of your technique? What about measurements of features $<10 \mu \mathrm{m}$ in size? The high resolution you use would allow this. Are there no significant changes on this size scale?

Authors: A $10 \mu \mathrm{m}$ voxel size does not allow the distinction between calcified cartilage and subchondral bone in the femoral condyle and the tibial plateau of the mice. Additionally, it is not possible to clearly delineate the non-calcified cartilage. Thus, no quantification of the thickness of both layers is feasible. With a $5 \mu \mathrm{m}$ voxel size, the non-calcified cartilage can be distinguished from the surrounding, however not when the thickness of the layer is smaller than $15 \mu \mathrm{m}$. Fig. $5 \mathrm{~d}$ shows that for the Sham group, where the average non-calcified cartilage thickness is well above $15 \mu \mathrm{m}$ (Fig. 5a), only $2.4 \pm 0.3 \%$ of the non-calcified cartilage thickness is below $15 \mu \mathrm{m}$. For this group, the $5 \mu \mathrm{m}$ voxel size images might be sufficient for 3D quantification. However, when severe lesions occur, such as in the case of the DMM group, about $14.5 \pm 7.7 \%$ of the non-calcified cartilage thickness is below $15 \mu \mathrm{m}$, and even about $10.5 \pm 5.6 \%$ of the thickness is below $10 \mu \mathrm{m}$. In the latter case, the $5 \mu \mathrm{m}$ voxel size images will not be able to capture important differences, and only the $2 \mu \mathrm{m}$ voxel size attainable with $\mathrm{CE}$-nanoCT is sufficient. 